General Relativity and Gravitation, Vol. 31, No. 07 (1999), 1015-1030

\title{
Decoherence in the Starobinsky Model
}

\author{
S.Biswas ${ }^{* a), b)}$, A.Shaw ${ }^{* * a)}$ and B.Modak ${ }^{a)}$ \\ a) Department of Physics, University of Kalyani, West Bengal, \\ India, Pin.- 741235 \\ b) IUCAA, Post bag 4, Ganeshkhind, Pune 411 007, India \\ * e-mail : sbiswas@klyuniv.ernet.in \\ ** e-mail : amita@klyuinv.ernet.in
}

\begin{abstract}
Starobinsky described an inflationary scenario in which quantum corrections to vacuum Einstein equations drive the inflation. The quantum cosmology of the model is studied by solving the Wheeler-DeWitt equation. A connection between uncertainty requirement, randomness in initial states and curvature fluctuation is studied with a Schrödinger-type equation through a time parameter prescription. The result obtained is applied to understand the decoherence mechanism in quantum gravity in the Starobinsky description.
\end{abstract}

KEY WORDS : Fourth-order gravity

\section{Introduction}

The Starobinsky scenario describes inflation through self-consistent solution of vacuum Einstein equations,

$$
R_{\mu \nu}-\frac{1}{2} g_{\mu \nu} R=-8 \pi G<T_{\mu \nu}>
$$

where $\left\langle T_{\mu \nu}>\right.$ is the expectation value of the energy momentum tensor. With a metric in Robertson-Walker form ,

$$
d s^{2}=d t^{2}-a^{2}(t) d \sigma_{k}^{2},
$$


where $d \sigma_{k}{ }^{2}$ is the metric on a unit three sphere for $k=1$, and the quantum corrections take a particularly simple form for free, massless, conformally invariant scalar fields:

$$
<T_{\mu \nu}>=k_{1}{ }^{(1)} H_{\mu \nu}+k_{3}{ }^{(3)} H_{\mu \nu}
$$

where $k_{1}$ and $k_{3}$ are constants and

$$
\begin{gathered}
(1)_{H \mu \nu}=2 R_{; \mu ; \nu}-2 g_{\mu \nu} R_{; \sigma}^{; \sigma}+2 R R_{\mu \nu}-\frac{1}{2} g_{\mu \nu} R^{2} \\
(3)_{H \mu \nu}=R_{\mu}{ }^{\sigma} R_{\nu \sigma}-\frac{2}{3} R R_{\mu \nu}-\frac{1}{2} g_{\mu \nu} R^{\sigma \tau} R_{\sigma \tau}+\frac{1}{4} g_{\mu \nu} R^{2}
\end{gathered}
$$

The characteristic features of the model are as follows:

(i) The model has a trace anomaly. Identifying

$$
8 \pi G<T_{\mu \nu}>=H_{o}^{-2(3)} H_{\mu \nu}+\frac{1}{6} M_{o}^{-2(1)} H_{\mu \nu}
$$

we get

$$
8 \pi G<T_{\nu}^{\nu}>=H_{o}{ }^{-2}\left(\frac{1}{3} R^{2}-R_{\nu \sigma} R^{\nu \sigma}\right)-M_{o}^{-2} R_{; \nu}^{; \nu}
$$

though $\left\langle T_{\nu}{ }^{\nu}\right\rangle$ vanishes for the classical conformally-invariant fields.

(ii) The model shows de-Sitter solutions

$$
\begin{aligned}
& a(t)=H_{o}^{-1} \cosh \left(H_{o} t\right), \quad k=+1 \\
& a(t)=a_{o} \exp \left(H_{o} t\right), \quad k=0 \\
& a(t)=H_{o}^{-1} \sinh \left(H_{o} t\right) \quad k=-1
\end{aligned}
$$

These solutions describe an inflationary phase driven entirely by quantum corrections.

(iii)Studying instability it has been found [1] that the solutions (8) - (10) are unstable under small perturbations and lead to a matter dominated universe at late times with $a(t) \propto t^{\frac{2}{3}}$.

(iv) The curvature fluctuations are found to be

$$
\left(\frac{\delta R}{R_{o}}\right)^{2} \sim \frac{G H_{o}{ }^{2}}{\pi} .
$$

The present work investigates the quantum cosmology of the Starobinsky model. Vilenkin [2] attempted this problem in order to study tunneling from "nothing" to the Starobinsky inflationary phase. The quantum analysis is then used to calculate the curvature fluctuations and assume a tunneling ansatz for the classical evolution of the model.

In view of recent trends in solving the Wheeler-DeWitt equation [3-6], we take up study of the Starobinsky description again to understand the curvature fluctuations, quantum force driving the Starobinsky inflation, initial conditions for the classical 
evolution from quantum phase. We also study the quantum to classical transition through the mechanism of decoherence.

It is worthwhile pointing out that, at the semiclassical level, the Starobinsky inflationary scenario has been criticized [7] on the ground that the inflationary solutions are not perturbatively expandable in the parameter of "quantum corrections" terms in the equation of motion or Lagrangian. There is also some question of treating the high order terms in the Lagrangian on an equal footing with the Einstein terms. Even then, studying the quantum cosmology of the Starobinsky description from the standpoint of quantum to classical transition would help us rethink the criticism of the model. Leaving aside this fact, it is instructive to look at the model as a toy example, to understand the current boundary condition proposals, decoherence mechanism and also the origin of quantum force in the early universe. Another motivation for studying the Starobinsky description is due to its inbuilt mechanism leading to spontaneous nucleation of the universe. Among the various types of inflationary models, the choice of the inflaton potential has no microscopic origin, i.e. the choice of potential is not dictated from particle physics phenomenology (except Coleman-Weinberg potential). For this reason, the warm inflationary scenario [8] has been a crazy inclusion among the various types of inflationary models. In view of this fact, studying of the quantum cosmology of the Starobinsky model would be interesting, at least from the viewpoint of quantum to classical transition with a view to understand the initial conditions of the pre-inflationary era.

In section II we obtain an approximate form of action for Starobinsky's description thereby obtaining the Wheeler-DeWitt equation, and discuss some results of Vilenkin [2] for comparison with our work. In section III we discuss the time prescription in quantum gravity to reduce the Wheeler-DeWitt equation to a Schroedinger form containing time. The initial conditions are then treated in the light of our discussion. In section IV, we mainly concentrate on the decoherence mechanism to understand the quantum to classical transition and this constitutes the main text of the present paper. In this paper we work with wormhole-dominance proposal [9] as a boundary condition to the Wheeler-DeWitt equation and find that Gaussian ansatz is the corresponding initial condition for the Schrödinger-Wheeler-DeWitt equation. This correspondence is a breakthrough in the present trend of investigation along these lines $[5,6]$. Lastly, we end up with a discussion in section V.

\section{Starobinsky Description}

The evolution equation for the scale factor is obtained from (1),(4), and (5) for $k=0$ and is given by

$$
H^{2}\left(H^{2}-H_{o}{ }^{2}\right)=\frac{H_{o}{ }^{2}}{M_{o}{ }^{2}}\left(2 H \ddot{H}+6 H^{2} \dot{H}-\dot{H}^{2}\right)
$$


In $(12) H=\frac{\dot{a}}{a}$ and the dot denotes the derivative with respect to cosmological time, and

$$
H_{o}=\left(8 \pi k_{3} G\right)^{-\frac{1}{2}}, M_{o}=\left(48 \pi k_{1} G\right)^{-\frac{1}{2}} .
$$

Equation (12) gives de Sitter solution $H=H_{0}$. Equation (12) for the condition $\dot{H}<H^{2}, \ddot{H}<<H \dot{H}$ reduces to

$$
H^{2}-H_{o}^{2}=6\left(\frac{H_{o}{ }^{2}}{M_{o}{ }^{2}}\right) \dot{H}
$$

with the solution

$$
H=H_{o} \tanh \left(\gamma-\frac{M_{o}^{2} t}{6 H_{o}}\right)
$$

where $\gamma=\frac{1}{2} \ln \left(\frac{\left(H(o)-H_{o}\right)}{H_{o}}\right)$. Long inflation requires

$M_{o}^{2}<<6 H_{o}^{2}$. For $H<<H_{o}$ the term proportional to $H^{4}$ in (12) can be neglected.

$$
2 H \ddot{H}+6 H^{2} \dot{H}-\dot{H}^{2}+M_{o}^{2} H^{2}=0,
$$

with an approximate solution

$$
H \simeq \frac{4}{3 t} \cos ^{2}\left(\frac{M_{o} t}{2}\right)\left[1-\frac{\sin M_{o} t}{M_{o} t}\right]
$$

Changing the origin of the time coordinates $M_{o} t \rightarrow\left(M_{o} t+\alpha\right)$, we get

$$
a(t)=\text { const. } t^{\frac{2}{3}}\left[1+\left(\frac{2}{3 M_{o} t}\right) \sin M_{o} t\right] .
$$

The expansion rate averaged over the oscillations period is

$$
\bar{H}=\frac{2}{3 t}
$$

so that $\bar{a} \propto t^{\frac{2}{3}}$ corresponds to the matter dominated universe.

We have carried out [10] numerical integration of (1) with a contribution from particle production. We found that for a late time behaviour showing a radiation dominated (and also for a matter dominated) evolution, the initial state emerges mostly with a deSitter phase. Solutions (out of various solutions) with $a(t)=a_{\text {min }}$ at $t=0$ greatly effects both the initial and late time behaviour. This numerical result suggests that the classical universe sits for infinite time at the turning point $a=a_{\text {min }}$, but quantum mechanically it enters into the disallowed region $a<a_{\min }=H_{o}^{-1}$ and tunnels out again in the classical region with a curvature fluctuation $\sim \frac{1}{a_{m i n}{ }^{2}} \sim$ $H_{o}^{2}$. To understand the quantum entanglement in the classical region, we need a quantum analysis in minisuperspace description. We need an action for the quantum description to get the Wheeler-DeWitt equation. For Starobinsky's description, (1) 
or (12), no closed form of action is available. However for $M_{o}<<H_{o}^{2}$, a closed form of action is obtained in the form

$$
S(R)=\frac{24 \pi}{G}\left(\frac{1}{R}+\frac{1}{6 M_{o}^{2}}+\frac{1}{R_{o}} \ln \frac{R}{R_{o}}\right)
$$

with

$$
L(R)=\frac{1}{16 \pi G}\left(R+\frac{R^{2}}{6 M_{o}^{2}}+\frac{R^{2}}{R_{o}} \ln \frac{R}{R_{o}}\right)
$$

where $R_{o}=12 H_{o}^{2}$. The curvature fluctuation is

$$
\begin{aligned}
\left(\frac{\delta R}{R_{o}}\right)^{2} & \sim \frac{2}{R_{o}^{2}\left|S^{\prime \prime}\left(R_{o}\right)\right|} \\
& \sim \frac{G H_{o}^{2}}{\pi}
\end{aligned}
$$

as expected.

To obtain the Wheeler-DeWitt equation through canonical quantization, one needs to determine the Hamiltonian $H$ to obtain the equation

$$
H \psi=0
$$

In view of higher order terms like $R^{2}$ and $R^{2} \ln \frac{R}{R_{o}}$ one gets

$$
S=\int \mathcal{L}(a, \dot{a}, \ddot{a}) d t
$$

in which the second derivatives $\ddot{a}$ cannot be removed from (24). To eliminate $\ddot{a}$ one writes

$$
S=\int \mathcal{L}(a, \dot{a}, R, \dot{R}) d t
$$

and takes

$$
S=2 \pi^{2} \int\left\{L(R) a^{3}-\beta\left[R-6 a^{-2}(1+\dot{a}+a \ddot{a})\right]\right\} d t .
$$

Varying $S$ with respect to $R$, we find

$$
\beta=a^{3} L^{\prime}(R)
$$

Putting (27) in (26) one can remove $\ddot{a}$ through integration by parts. Introducing new variables $q$ and $x$ instead of $a$ and $R$,

$$
\begin{gathered}
q=H_{o} a\left(\frac{L^{\prime}}{L_{o}^{\prime}}\right)^{\frac{1}{2}} \\
x=\frac{1}{2} \ln \left(\frac{L^{\prime}}{L_{o}^{\prime}}\right)
\end{gathered}
$$


and using the condition $M_{o}^{2}<<H_{o}^{2}$ one obtains the Wheeler-DeWitt equation with the replacement $P_{q} \rightarrow-i \frac{\partial}{\partial q}, P_{x} \rightarrow-i \frac{\partial}{\partial x}$ as

$$
\left[\frac{\partial^{2}}{\partial q^{2}}-\frac{1}{q^{2}} \frac{\partial^{2}}{\partial x^{2}}-V(q, x)\right] \psi(q, x)=0
$$

$\operatorname{In}(30)$

$$
\begin{aligned}
x & =\frac{1}{2} \ln \left(\frac{R}{R_{o}}\right) \\
V(q, x) & =\lambda^{-2} q^{2}\left(1-q^{2}+\mu^{2}(x) q^{2}\right) \\
\mu^{2}(x) & =\frac{M_{o}^{2}}{2 H_{o}{ }^{2}}\left(2 x+e^{-2 x}-1\right)
\end{aligned}
$$

where

$$
\lambda=\frac{G M_{o}^{2}}{6 \pi} .
$$

We make the transformation $Q=\frac{q}{\sqrt{\lambda}}$, in $(30)$ and get

$$
\left[\frac{\partial^{2}}{\partial Q^{2}}-\frac{1}{Q^{2}} \frac{\partial^{2}}{\partial x^{2}}-Q^{2}\left(1-Q^{2} U(x)\right)\right] \psi=0
$$

where

$$
U(x)=\lambda\left(1-\mu^{2}(x)\right) .
$$

Vilenkin obtained a solution of (35) in the region $q>q_{x}$, where $q=q_{x} \simeq 1+\frac{1}{2} \mu^{2}(x)$ is the turning point,

$$
\psi=\pi^{\frac{1}{2}}\left|V_{o}(q)\right|^{-\frac{1}{4}} \exp \left\{-\frac{1}{3 \lambda}\left(1+\mu^{2}(x)\right)\left[1-i\left(q^{2}-q^{2} \mu^{2}-1\right)^{3 / 2}\right]+\frac{i \pi}{4}\right\}
$$

where

$$
V_{o}(q)=\lambda^{-2} q^{2}\left(1-q^{2}\right)
$$

We take $q>>1$ in (37) and find

$$
\begin{aligned}
\psi & \simeq \pi^{\frac{1}{2}} \lambda^{\frac{1}{2}} q^{-1} \exp \left\{-\frac{1}{3 \lambda}\left(1+\mu^{2}(x)\right)\left[1-i q^{3}\left(1-\mu^{2}(x)\right)^{3 / 2}\right]+\frac{i \pi}{4}\right\} \\
& \simeq(\pi \lambda)^{\frac{1}{2}} q^{-1} \exp \left\{-\frac{1}{3 \lambda}\left(1+\mu^{2}(x)\right)\left[1-i q^{3}\left(1-\mu^{2}(x)\right)^{3 / 2}\right]\right\} .
\end{aligned}
$$

For $x<<1$,

$$
|\psi|^{2} \propto \exp \left[-\frac{4 \pi x^{2}}{G H_{o}^{2}}\right] .
$$

If one temporarily sets aside the normalization restriction on $\psi$,

$$
<\left(\frac{\delta R}{R_{o}}\right)^{2}>=4<x^{2}>\sim \frac{G H_{o}^{2}}{2 \pi},
$$


in qualitative agreement with the classical result. The fluctuation in expansion rate is

$$
\delta_{o} \equiv \frac{\delta H}{H_{o}}=\frac{1}{2} \frac{\delta R}{R_{o}} \sim\left(\frac{G H_{o}^{2}}{8 \pi}\right)^{\frac{1}{2}} .
$$

This $\delta_{o}$ determines the duration [see (15)] of the inflationary phase in the model

$$
t_{*} \sim\left(\frac{3 H_{o}}{M_{o}^{2}}\right) \ln \frac{2}{\delta_{o}} .
$$

Taking $H_{o} \sim 0.7 m_{p}$ and using (42) and (43) we get $\delta_{o} \sim 0.14, t_{*} \sim 8 \frac{H_{o}}{M_{o}^{2}}$. Using $8 \pi k_{1}=1.8$ (restriction from $S U(5)$ model) and the value of $M_{o}$ from (13), $t_{*} \sim$ $4 \times 10^{10} H_{o}^{-1}$. This value of $t_{*}$ is more than sufficient to solve the horizon and flatness problem. The results quoted so far are standard and will be needed to understand much of what follows.

\section{Reduction to Schrödinger Form}

Recent trends in quantum cosmology [3-6] suggest that the solution of (35) should be written as

$$
\psi(Q, x)=\exp [i S(Q)] \Phi(Q, x)
$$

in which WKB action $S$ depends only on $Q$. However the philosophy behind this reduction though differs, most of the workers obtained Schroedinger equation through a prescription on time variable. Apart from the interpretation, almost everybody remains silent about the normalization, and consequently also about the probability concept. These two concepts are of utmost importance for the interpretational framework of the wavefunctional $\psi(Q, x)$. We have shown [9] that if one takes contribution from wormholes in WKB ansatz, the wavefunctional becomes normalizable. When boundary conditions corresponding to 'tunneling' and 'no boundary' proposals are introduced, one recovers the respective wavefunction. We have shown elsewhere that if one introduces the probabilistic concept through a continuity-like equation which also gives a time parameter prescription, the wavefunctional $\psi(Q, x)$ separates into the form (44) provided the classical Hamilton-Jacobi equation remains satisfied. We have thus a dynamical content to the form (44). We report briefly here the results only $[11,12]$. We put (44) in (35) and use

$$
\frac{\partial}{\partial t}=-\frac{1}{Q} \frac{\partial S}{\partial Q} \frac{\partial}{\partial Q}
$$

and we find

$$
i \frac{\partial \Phi}{\partial t}=\left[-\frac{1}{2 Q^{3}} \frac{\partial^{2}}{\partial x^{2}}+\frac{Q^{3}}{2} U(x)+\frac{1}{2 Q}\right] \Phi .
$$

Remembering that $\frac{\partial^{2}}{\partial Q^{2}}$ in (35) remains multiplied by a factor $\hbar^{2}$ and $S$ in $(44) \sim \frac{S}{\hbar}$, we neglected terms $\frac{\partial^{2} \Phi}{\partial Q^{2}}$ which are of the order of $\hbar^{2}$. It may be noted that our 
prescription does not violate unitarity which is obtained in the other prescription. The assumption (45) is consistent with (35) as well as with the Einstein classical equation. Equation (46) is the time dependent Schroedinger equation in quantum gravity.

To understand the salient features of the Starobinsky description, let us proceed with the solution of (46) with Gaussian ansatz

$$
\Phi(Q, x)=N(t) e^{-\frac{\Omega(t)}{2} x^{2}}
$$

Substituting (47) in (46) we get coupled equations

$$
\begin{gathered}
i \frac{d}{d t} \ln N=\frac{\Omega}{Q^{3}}+Q^{3} \lambda+\frac{1}{Q} \\
i \dot{\Omega}=\frac{\Omega^{2}}{Q^{3}}+\frac{M_{o}^{2} \lambda}{H_{o}^{2}} Q^{3} .
\end{gathered}
$$

With the anstaz

$$
\Omega=-i Q^{3} \frac{\dot{y}}{y}
$$

one finds from (49)

$$
\ddot{y}+3 \frac{\dot{Q}}{Q} \dot{y}-\frac{\lambda M_{o}^{2}}{H_{o}^{2}} y=0 .
$$

Introducing conformal time coordinate $\eta$ according to $d t=Q d \eta$, one finds

$$
y^{\prime \prime}+2 \frac{Q^{\prime}}{Q} y^{\prime}-\frac{\lambda M_{o}^{2} Q^{2}}{H_{o}^{2}} y=0
$$

where prime denotes a derivative with respect to $\eta$. With the view that the classical model has a de Sitter solution, we take $Q(\eta)=-\frac{1}{\sqrt{\lambda} \eta}$, where $\eta$ runs from $-\infty$ to 0 . Equation (52) now reads

$$
y^{\prime \prime}-\frac{2}{\eta} y^{\prime}-\frac{M_{o}^{2}}{H_{o}^{2} \eta^{2}} y=0
$$

which is solved by

$$
y=\eta^{3 / 2 \pm \sqrt{9 / 4+m^{2}}} .
$$

where we take $m^{2}=\frac{M_{0}^{2}}{H_{0}{ }^{2}}$. In the limit $m^{2}<<\frac{9}{4}$ (which is usually assumed to be satisfied in the inflationary model and in our description) such that

$$
\sqrt{9 / 4+m^{2}} \sim\left(3 / 2+m^{2} / 3\right)
$$

In the conformal time, the expression (50) reads

$$
\Omega=-i Q^{2} \frac{y^{\prime}}{y}
$$


so that using (54) and (55) one finds

$$
\Omega=-i m^{2} \sqrt{\lambda} \frac{Q^{3}}{3} .
$$

As $\Omega$ is imaginary the state (47) is not normalizable. To study decoherence one needs a real part in (57). We take up decoherence in the next section.

\section{Decoherence mechanism}

Decoherence is a mechanism through which we understand how the classical world arises from a quantum wavefunction of the universe. In decoherence, quantum interference effects are suppressed by the averaging out of microscopic variations not distinguished by the associated observables. In standard quantum theory this is referred to as the 'collapse of the wavefunction'. It is a formidable task to forbid the occurrence of linear superposition of states localized in far away spatial regions and induce an evolution agreeing with classical mechanics. In the context of quantum gravity the situation further complicates due to the absence of "time" because the Wheeler DeWitt equation when compared to Schrödinger equation

$$
i \hbar \frac{\partial \psi}{\partial t}=H \psi
$$

gives a timeless character to the wavefunction of the universe. Though we recovered the form (58) with a reduced Hamiltonian given in (46), it must be ensured that during the quantum to classical transition, none of the successful quantitative predictions of the inflationary scenario for the present day universe is changed. An important aspect in this direction is to choose an initial condition for eqs. (58) or (35) such that basic input "inflation" remains undisturbed in the description. With respect to the Wheeler-DeWitt equation, the wavefunction $\psi$ is described with some boundary condition proposals namely (i) Hartle-Hawking [13] (ii) Vilenkin [14] and (iii) Wormhole-Dominance proposal [9]. The latter one is recently proposed by us.

Now we will show that an initial adiabatic ground state with a Gaussian form is a suitable choice provided the quantum cosmological initial conditions correspond to the wormhole dominance proposal, or at least to the Hartle-Hawking proposal. This would also justify the correct choice of the boundary conditions. Using (57) in (48), we obtain for large $Q$

$$
N=N_{o} \exp \left[-\frac{i Q^{3} \sqrt{\lambda}}{3}\right] .
$$

The constant $N_{0}$ will be evaluated through the wormhole dominance proposal. The wavefunction now reads, corresponding to (46) or (58),

$$
\psi=N_{o} \exp \left[-\frac{i Q^{3} \sqrt{\lambda}}{3}+i \frac{M_{o}^{2} \sqrt{\lambda} Q^{3}}{6 H_{o}^{2}} x^{2}\right] .
$$


We write the exponent in (60) as

$$
\begin{aligned}
S & =-\frac{i Q^{3} \sqrt{\lambda}}{3}\left(1-\frac{1}{2} \frac{M_{o}^{2}}{H_{o}^{2}} x^{2}\right) \\
& =-\frac{i}{3}\left[Q^{2} \lambda\left(1-\frac{M_{o}^{2}}{H_{o}^{2}} x^{2}\right)\right]^{3 / 2} \frac{1}{\lambda\left(1-\frac{M_{o}^{2}}{H_{o}^{2}} x^{2}\right)}
\end{aligned}
$$

since $\frac{M_{o}^{2}}{H_{o}^{2}} x^{2}<<1$. Comparing with (33) we write (61)

$$
\begin{aligned}
S & =-\frac{i}{3 \lambda\left(1-\mu^{2}(x)\right)}\left[\lambda Q^{2}\left(1-\mu^{2}(x)\right)\right]^{3 / 2} \\
& =-\frac{i}{3 \lambda\left(1-\mu^{2}(x)\right)}\left[q^{2}\left(1-\mu^{2}(x)\right)\right]^{3 / 2} \\
& \simeq-\frac{i}{3 \lambda}\left(1+\mu^{2}(x)\right)\left[q^{2}\left(1-\mu^{2}(x)\right)-1\right]^{3 / 2} .
\end{aligned}
$$

The last step will be justified from the Wheeler-DeWitt equation itself since $q>>1$. The wormhole dominance proposal considers incorporating the repeated reflections from the turning points $q=o$ and $q=q_{x}=\frac{1}{\left(1-\mu^{2}(x)\right)^{\frac{1}{2}}}$ to contribute to $N_{o}$ as

$$
N_{o}=\frac{\exp S\left(q_{x}, 0\right)}{1-\exp \left[2 S\left(q_{x}, 0\right)\right]}
$$

Here

$$
S\left(q_{x}, 0\right) \equiv|S|_{0}^{q_{x}}
$$

Evaluating (63) we find

$$
N_{o}=\frac{\exp \left\{\frac{1}{3 \lambda}\left(1+\mu^{2}(x)\right)\right\}}{\left(1-\exp \left\{2 / 3 \lambda\left(1+\mu^{2}(x)\right)\right\}\right)}
$$

Hence

$$
\psi=\frac{\exp \left[\frac{1}{3 \lambda}\left(1+\mu^{2}(x)\right)\left\{1-i\left(q^{2}\left(1-\mu^{2}(x)\right)-1\right)^{\frac{3}{2}}\right\}\right]}{\left(1-\exp \left\{2 / 3 \lambda\left(1+\mu^{2}(x)\right)\right\}\right)} .
$$

We continue $(66)$ in the region $q^{2}\left(1-\mu^{2}\right)<1$ to get

$$
\psi=\frac{\exp \left\{\frac{1}{3 \lambda}\left(1+\mu^{2}(x)\right)\left[1-\left(1-q^{2}+q^{2} \mu^{2}\right)^{3 / 2}\right]\right\}}{\left(1-\exp 2 / 3 \lambda\left(1+\mu^{2}(x)\right)\right)} .
$$

If we leave aside the denominator of (67), the wavefunction (67) corresponds to the Hartle-Hawking wavefunction.

Thus the initial condition for the Schrödinger-Wheeler-DeWitt equation (46) turns out to be that at an early time (near the onset of inflation), the modes are in their adiabatic ground state due to wormhole dominance and this serves as the initial 
conditions on the Wheeler-DeWitt wavefunction. Unlike the claim by some authors, it also establishes that Hartle-Hawking initial conditions also provides inflation and serve as a seed for decoherence to suppress the interference for quantum to classical transition. One important feature of (67) is that at $q \rightarrow 0,(67)$ reduces to

$$
\psi \sim \frac{\exp \left\{\frac{q^{2}}{2 \lambda}\right\}}{\left(1-\exp \left\{2 / 3 \lambda\left(1+\mu^{2}(x)\right)\right\}\right)},
$$

characteristic of the Hartle-Hawking wavefunction. As is evident from (34), $\lambda$ is a small quantity, and hence, considering the denominator of (68), we find

$$
|\psi|^{2} \sim e^{-\frac{1}{3 \lambda}\left(1+\mu^{2}(x)\right)}
$$

This gives the curvature fluctuation as in (41).

The steps leading to the form (68) from (60) indicates that at the very early stage (within the turning points) the quantum superposition principle has been effective such that the time has lost its meaning. The interference between $e^{i S}$ and $e^{-i S}$ is maintained through the wormholes that act as a driving quantum force. We have shown elsewhere [15] that without any reference to the Wheeler-DeWitt equation one can also recover eq. (46) exactly using only the classical Einstein-Hamilton-Jacobi equation with a directional time derivative

$$
\frac{\partial}{\partial t}=-\frac{1}{Q} \frac{\partial S_{o}}{\partial Q} \frac{\partial}{\partial Q}
$$

i.e., the scale factor (gravitational field) itself acts as time. Just at the classical turning point, time begins to flow and the effect of the quantum turning point (at which the wormhole contributes) only survives through $N(t)$ in the Gaussian ansatz i.e., the states $e^{i S}$ and $e^{-i S}$ begin to decohere. It has been guessed by some authors [16] that some sort of boundary conditions at small scales may lead to quantum effects in the vicinity of the turning point. The 'wormhole dominance' proposal exactly establishes this aspect, keeping the necessary coinage for inflation. If we are to bridge the solution (47) with (67), the reduction

$$
S=S_{o}(Q)+S_{1}(Q, x)
$$

with

$$
\psi=\exp \left[i S_{1}(Q, x)\right]
$$

is an unavoidable fact. Kiefer [5] named this reduction as the 'relevant' and the 'environmental' degrees of freedom. The solution (67) refers to the ground state wavefunction. The higher degrees of freedom ('higher multipoles') when taken into account, eq. (53) modifies to

$$
y^{\prime \prime}-\frac{2}{\eta} y^{\prime}+\left(n^{2}-\frac{M_{o}^{2}}{H_{o}^{2} \eta^{2}}\right) y=0 .
$$


Evaluating $\Omega$ along the lines of $[5]$ we find

$$
\Omega \simeq \frac{n^{2} Q^{2}}{n^{2}+Q^{2} H^{2}}\left(n+i Q H_{o}\right)-i \frac{M_{o}^{2} Q^{3}}{3 H_{o} \lambda},
$$

and the decoherence factor responsible to suppress interference terms is written as

$$
\begin{aligned}
\exp \left(-D_{ \pm}\right) & =\exp \left(-\frac{1}{4} \operatorname{Tr} \frac{\Omega_{I}^{2}}{\Omega_{R}^{2}}\right) \\
& =\exp \sum_{n} n^{2}\left(\frac{\Omega_{I}}{\Omega_{R}}\right)^{2} .
\end{aligned}
$$

We choose the adiabatic vacuum state with positive frequency as the solution of (71) or (53) and this acts as initial vacuum state. This initial state evolves at $\eta \rightarrow+\infty$ according to a WKB form,

$$
y=\frac{\alpha}{\sqrt{2 \omega}} e^{i \int^{\eta} \omega\left(\eta^{\prime}\right) d \eta^{\prime}}+\frac{\beta}{\sqrt{2 \omega}} e^{-i \int^{\eta} \omega\left(\eta^{\prime}\right) d \eta^{\prime}},
$$

where $\alpha$ and $\beta$ are the usual Bogolubov co-efficients. For the WKB state (74) one finds

$$
\begin{aligned}
\Omega & \simeq \omega+\frac{i \dot{\omega}}{2 \omega} \\
& =\sqrt{n^{2}-m^{2} Q^{2}}-i \frac{m^{2} Q Q^{\prime}}{2\left(n^{2}-m^{2} Q^{2}\right)}
\end{aligned}
$$

with $m^{2}=\frac{M_{o}^{2}}{H_{o}^{2}} \lambda$. Hence

$$
D_{ \pm}=\frac{m^{4} Q^{2} Q^{\prime 2}}{8} \sum_{n} \frac{n^{2}}{\left(n^{2}-m^{2} Q^{2}\right)^{3}} .
$$

Using

$$
\frac{1}{\zeta}-\pi \cot \pi \zeta=\sum_{1}^{\infty} \frac{2 \zeta}{n^{2}-\zeta^{2}}
$$

and after some straightforward manipulation we find

$$
D_{ \pm}=\frac{\pi m^{3} Q Q^{\prime 2}}{128}\left[\frac{\pi}{m Q}\left(1+\cot ^{2} \pi m Q\right)+\frac{\cot \pi m Q}{(m Q)^{2}}-2 \pi^{2} \cot \pi m Q\left(1+\cot ^{2} \pi m Q\right)\right] \text {. }
$$

In the limit $m Q>>1$, one finds

$$
\exp \left(-D_{ \pm}\right) \simeq \exp \left(-\frac{\pi m Q^{\prime 2}}{128 Q}\right)=\exp \left(-\frac{\pi m H_{o}^{2} Q^{3}}{128}\right)
$$

This result is also obtained by Kiefer [6]. For $m \simeq 100 \mathrm{GeV}, H_{o} \simeq 55 \mathrm{~km} / \mathrm{sec} M p c$, and $Q \simeq H_{o}^{-1}$, this is

$$
\exp \left(-10^{+43}\right) \simeq 0
$$

Hence decoherence is efficient for large $Q$ even in the Starobinsky description. This argument leads us to reconsider the Starobinsky description despite the criticisms labelled against it. 


\section{Discussion}

The Starobinsky model provides a viable description both in the classically allowed and in the forbidden region. It is a model where one loop quantum corrections spontaneously allow the universe to make the transition to a deSitter phase and then to a classical Friedmann stage with a small perturbation. It is a model of spontaneous nucleation rather than inflation-driven inflation. In our previous work [9] we achieved the advantage of normalized wavefunction with probabilistic interpretation but there still remains no certainty about the adoption of a particular boundary condition proposal. Within the perspective of the Schroedinger-Wheeler-DeWitt equation describing the time evolution of the universe, we found that Hawking type boundary conditions definitely establish inflation (since we take $Q=-\frac{1}{\sqrt{\lambda} \eta}$ ), as the characteristic feature of Starobinsky model, unlike the claim by some workers in favour of the tunneling proposal. The wormhole dominance proposal serves as an initial condition for decoherence to effect quantum to classical transition, because the Gaussian ansatz for SWD equation (valid in the classically allowed region) leads, after continuation, to the wavefunction according to the wormhole dominance proposal. We observe that the Starobinsky model (basically reminding us of a $R^{2}$-cosmology) is equivalent to an Einstein gravity plus a scalar field and this inflaton-like scalar field arises from one loop quantum corrections. It may be mentioned that quantum cosmology with Hartle-Hawking boundary conditions for a model Einstein gravity plus a dialaton was studied by Okada and Yoshimura [17]. They briefly comment that the nucleation of the classical universe would be exponentially suppressed if one takes the tunneling proposal. The quantum nature of the early universe thus guarantees the universal validity of superposition principle. This in turn incorporates multiple reflections between the turning points. The wormhole dominance proposal takes this fact justifiably defining a normalization constant on the basis of superposition principle favouring probabilistic interpretation rather than the concept of 'conditional probability'. In our previous work [9] we have shown that the normalization constant prescription in the wormhole dominance proposal is equivalent to the contribution

of wormholes leading to an interpretational framework for wormhole picture (lack of which created a confusion about its introduction), apart from Klebanov and Coleman's arguments, as a driving quantum force in the early universe. The ensemble of quantum universe models would thus be a realistic situation in quantum gravity.

\section{Acknowledgment}

A. Shaw acknowledges the financial support from ICSC World Laboratory, LAUSSANE, Switzerland during the course of the work.

\section{References}


1. Vilenkin A.,(1985), Phys. Rev. D32, 2511.

2. Vilenkin A.,(1983), Phys. Rev. D27, 2848.

3. Wada S., (1986), Nucl. Phys. B276, 729.

4. Kuchar K.V.,(1992), In Proc. of the fourth Canadian Conference on General Relativity and Relativistic Astrophysics, edited by G. Kunstatter, D. Vincent and J. Williams (World Scientific, Singapore), P 211-314.

5. Kiefer C., (1992), Phys. Rev. D45, 2044.

6. Kiefer C., (1992), Phys. Rev. D46, 1658.

7. Simon J.Z., (1992), Phys. Rev. D45, 1553

Parker L. and Simon J.Z., (1993), Phys. Rev. D47, 1339.

8. Berera A., (1997), Phys. Rev. D55, 3346 and ref. there in.

9. Biswas S., Modak B. and Biswas D., (1996), Phys. Rev. D55, 4673.

10. Shaw A., Biswas D., Modak B. and Biswas S., (1999) Pramana - J. Phys. 52, 1.

11. Biswas S., Shaw A. and Modak B.,(1998), "Time In Quantum Gravity", gr-qc/9906010.

12. Biswas S., Shaw A. and Biswas D., (1998), "Schroedinger Wheeler-DeWitt Equation In Multidimensional Cosmology", gr-qc/9906009.

13. Hawking S.W., (1984), Nucl. Phys. B239, 257

Hawking S.W. and Page D.N., (1986), Nucl. Phys. B264, 184.

14. Vilenkin A.,(1987), Phys. Rev. D37, 888.

15. Biswas S., Shaw A., Modak B. and Biswas D., (1998), "Quantum Gravity Equation In Schroedinger Form", gr-qc/9906011.

16. Conardi H.D. and Zeh H.D., (1991), Phys. Lett. A154, 321.

17. Okada Y. and Yoshimura M., (1986), Phys. Rev. D33, 2164. 